\title{
Development of a cold-neutron imaging detector based on thick gaseous electron multiplier
}

\author{
M. Cortesi, ${ }^{1,2}$ R. Zboray, ${ }^{1}$ A. Kaestner, ${ }^{1}$ and H.-M. Prasser ${ }^{2}$ \\ ${ }^{1}$ Paul Scherrer Institut (PSI), Villigen PSI CH-5234, Switzerland \\ ${ }^{2}$ Swiss Federal Institute of Technology (ETH), Zurich CH-8092, Switzerland
}

(Received 9 January 2013; accepted 7 February 2013; published online 26 February 2013)

\begin{abstract}
We present the results of our recent studies on a cold-neutron imaging detector prototype based on THick Gaseous Electron Multiplier (THGEM). The detector consists of a thin Boron layer, for neutron-to-charged particle conversion, coupled to two THGEM electrodes in cascade for charge amplification and a position-sensitive charge-readout anode. The detector operates in $\mathrm{Ne} /(5 \%) \mathrm{CF}_{4}$, at atmospheric pressure, in a stable condition at a gain of around $10^{4}$. Due to the geometrical structure of the detector elements (THGEM geometry and charge read-out anode), the image of detector active area shows a large inhomogeneity, corrected using a dedicated flat-filed correction algorithm. The prototype provides a detection efficiency of 5\% and an effective spatial resolution of the order of 1.3 mm. @ 2013 American Institute of Physics. [http://dx.doi.org/10.1063/1.4793225]
\end{abstract}

\section{INTRODUCTION}

Cold-neutron imaging provides radiographic (twodimensional) or tomographic (three-dimensional), spatially resolved images of the internal structure of bulk samples that are not accessible by other techniques. ${ }^{1-3}$ Its implementation has become increasingly popular in different branches of applied science. This is due to an ever-growing availability of strong neutron sources and to the developments of novel imaging detector technologies. Potential applications range from fundamental research to industry, including material test and quality-control inspection in metallurgy, ${ }^{4}$ fuel cells research, ${ }^{5}$ study of objects from cultural heritage, ${ }^{6,7}$ geoscience and soil physics, ${ }^{8,9}$ investigation of thermal hydraulic phenomena, ${ }^{10,11}$ homeland security, ${ }^{3}$ and imaging of biological specimens. ${ }^{3}$

Unlike charged particles (electrons, protons, alpha particles, etc.) cold-neutrons do not cause any direct ionization, making difficult their detection and posing a range of special problems related to the design, construction, and implementation of the detector technology. Instead, cold-neutron detection requires capture reactions, which released great amount of energy in the form of charge particles and/or photons.

The most common approach in digital neutron imaging implies the conversion of the neutron field information into visible light through a scintillation process. The neutroninduced light signals can be detected either by sensitive camera systems or by silicon $(\mathrm{Si})$ based photo-detectors including photodiodes, avalanche photodiodes (APDs), and Si drift detectors. These are well-established detection techniques that provide excellent spatial resolution down to $20-30 \mu \mathrm{m}$, though at the expense of a limited field of view. ${ }^{12}$ Although the frame rate of the camera, due to latest development in high speed scientific CMOS technology can be as high as a few hundred frames per second, highly dynamic process requiring kframes per second are still beyond reach. Imaging at such high rates might also come to the limit given by the brightness of the presently available neutron sources, unless a very coarse spatial resolution $(>0.5 \mathrm{~cm})$ is satisfactory for the given application. A recent innovative solution is represented by hybrid imaging detectors, comprising of micro-channel plates (MCPs) coupled to pixelated CMOS application specific integrated circuit (ASIC) chips (Timepix or Medipix) for charge readout. MCP consists of a regular array of tiny (micrometers) tubes made of glass mixed with small concentration of neutron converter substances. These devices are capable of high detection efficiency ( $>40 \%$ ), high counting rate $(>1 \mathrm{MHz}$ ), and good time resolution (few tens of nanoseconds); however they have limited active area. ${ }^{13}$

Very large active-area can be achieved by gaseous detectors. These devices use gas converter $\left({ }^{10} \mathrm{BF}_{3}\right.$ or $\left.{ }^{3} \mathrm{He}\right)$ or solid converter (typically ${ }^{10} \mathrm{~B}$ or ${ }^{6} \mathrm{Li}$ ) within the counting gas volume. The development of position-sensitive proportional counters has particularly led to imaging detector development for neutron-scattering applications, neutron beam monitor, neutron reflectometry, and diffraction. Gas converter detector cannot compete easily with sub-millimeter position resolution, because of the long capture product range; the best wire chamber detector resolution to date is about $400 \mu \mathrm{m}$, with limited counting rate capability. ${ }^{14}$ Better performances were achieved with the introduction of micro-pattern gaseous detector (MPGD). In this structure electron avalanche occurs in a narrow amplification region (typically $50-100 \mu \mathrm{m}$ ), with the benefits of a high rate capability $\left(>1 \mathrm{MHz} / \mathrm{cm}^{2}\right)$, excellent time resolution (few nanoseconds measured with UV-photon and relativistic electrons ${ }^{15}$ ), resistance to aging, and intrinsic ion and photon feedback suppression. For example, the Gas Electron Mulitplier (GEM) uses biased stacks of perforated kapton foils to amplify ionization electrons released in a small drift gas gap by the neutron-induced charged particle. ${ }^{16}$ For instance, in the CASCADE detector the GEM foils are used as substrate for the solid neutron converter $\left({ }^{10} \mathrm{~B}\right)$, permitting to cascade many converter layers in order to reach high detection efficiency. ${ }^{17}$

We develop various high-resolution instrumentation and measurement techniques for investigating various relevant industrial processes, like dynamic gas-liquid two-phase 
flows. ${ }^{18,19}$ As part of these activities, we have recently initiated an extensive study of two-phase flows in nuclear fuel bundle models by cold-neutron imaging at the Imaging with COld Neutrons (ICON) beam line on the SINQ spallation source, ${ }^{10,11}$ using an optical cold-neutron imaging system that includes a scintillator plate, a mirror, and a CCD camera. ${ }^{20}$ Though the optical system is capable of providing an excellent spatial resolution (around hundred micrometers), it is relatively an expensive device and it does not allow high-frame rates $(\mathrm{kHz})$ at large field of views $\left(>20 \times 20 \mathrm{~cm}^{2}\right)$.

In this work we discuss and present the development of an attractive solution for two-dimensional cold-neutron imaging detector, based on THick Gaseous Electron Multiplier (THGEM); ${ }^{21}$ it could be an alternative/complementary solution to the ICON optical system, for high frame-rate and large field of view imaging. The THGEM is a novel hole-type gaseous-avalanche multiplier, similar to the standard GEM, but it is more robust since it has about 5-20-fold expanded dimensions. It is manufactured economically by mechanically drilling sub-millimeter diameter holes, spaced by a fraction of $1 \mathrm{~mm}$, in a thin (generally a fraction of a $\mathrm{mm}$ ) printedcircuit board (PCB). The operation of THGEM in Ne-based mixtures at atmospheric pressure allows reaching high electron multiplication $\left(\sim 10^{6}\right.$ with two cascaded THGEMs) and high dynamic range, ${ }^{22}$ the latter is particularly crucial for applications with a highly ionizing radiation background and for applications with a wide spectrum of deposited energies. The performance of the imaging system in terms of detection efficiency, spatial resolution, and image quality have been determined irradiating the detector with cold neutrons at the ICON facility of the neutron spallation source SINQ (PSI) ${ }^{20}$ At the operational conditions used in our experiments (a very high $\mathrm{L} / \mathrm{D}=12083$; spectra and more technical details can be found in Ref. 20), the ICON beamline provided a white spectrum $(1-10 \AA)$ neutron flux of approximately $1.5 \times 10^{4}$ neutron/ $\mathrm{cm}^{2} / \mathrm{s}$.

\section{DETECTOR SETUP}

The schematic drawing of the detector is shown in Figure 1; it comprises of a ${ }^{10} \mathrm{~B}$-coated cathode electrode, used for cold neutrons to charge particles $\left({ }^{4} \mathrm{He}\right.$ and $\left.{ }^{7} \mathrm{Li}\right)$ conversion, coupled to a THGEM-based charge readout. The ${ }^{10} \mathrm{~B}$-coated cathode electrode was produced by CASCADE Detector Technology GmbH (CASCADE GmbH) and it consists of a $0.1 \mathrm{~mm}$ thick aluminum foil with one surface coated with a $1-\mu \mathrm{m}$ thick ${ }^{10} \mathrm{~B}$ layer (density $\sim 2.35 \mathrm{~g} / \mathrm{cm}^{3}$ ). The ${ }^{10} \mathrm{~B}$ layer covers an effective area of $100 \times 100 \mathrm{~mm}^{2}$. According to the manufacturer, the ${ }^{10} \mathrm{~B}$ layer has good thickness homogeneity, with a thickness variation below 5\%. The converter layer was glued on a $3 \mathrm{~mm}$ thick aluminum electrode and mounted above the first THGEM cascade as is shown in Figure 1. When an incident neutron interacts with ${ }^{10} \mathrm{~B}$, an alpha $\left({ }^{4} \mathrm{He}\right)$ particle and a lithium-7 $\left({ }^{7} \mathrm{Li}\right)$ ion are released according to

$$
\begin{aligned}
{ }^{10} \mathrm{~B}+\mathrm{n} \rightarrow & { }^{4} \mathrm{He}(1.47 \mathrm{MeV})+{ }^{7} \mathrm{Li}(0.84 \mathrm{MeV}) \\
& +\operatorname{gamma}(0.48 \mathrm{MeV}) \quad(94 \%),
\end{aligned}
$$

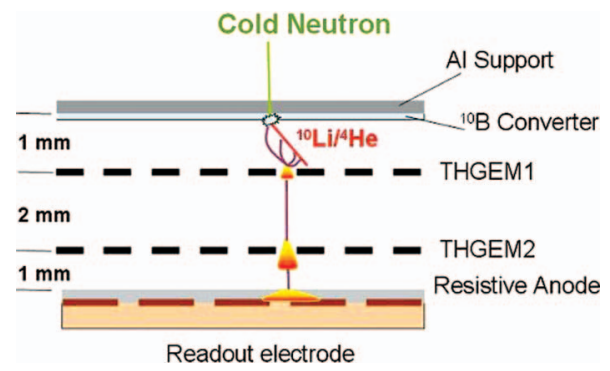

FIG. 1. Schematic drawing of the detector configurations: the THGEMbased readout was coupled to a 1- $\mu \mathrm{m}$ thick 10B layer deposited on an $\mathrm{Al}$ support for neutron-to-charged particle conversion.

$$
\begin{aligned}
{ }^{10} \mathrm{~B}+\mathrm{n} \rightarrow & { }^{4} \mathrm{He}(1.79 \mathrm{MeV})+{ }^{7} \mathrm{Li}(1 \mathrm{MeV}) \\
& +2.79 \mathrm{MeV} \quad(6 \%) .
\end{aligned}
$$

The ${ }^{4} \mathrm{He}$ and ${ }^{7} \mathrm{Li}$ particles escaping the converter lose part of their energy in the drift gap in the form of ionization electrons. Upon the action of suitable electric fields, these ionization charges are then directed towards and therein multiplied by two THGEM stages and localized by the position-sensitive charge readout. ${ }^{23}$ The latter is based on discrete delay-line position encoding, ${ }^{24}$ consisting of discrete inductor-capacitor (LC) filter-cells connected to individual cathode or anode strips (2 mm pitch) that run at orthogonal directions ( $\mathrm{x}$ and $y$ coordinate). Signals at the ends of the two delay lines are read by current amplifiers; charge localization is then computed from the propagation time of the induced signal traveling along the delay-line.

\section{RESULTS}

\section{A. Detection efficiency}

The detection efficiency has been determined by systematic computer simulations using $\mathrm{MCNP}^{25}$ and compared to experimental results. The simulations were designed to compute the neutron interaction rate in the $1 \mu \mathrm{m}{ }^{10} \mathrm{~B}$ layer and the escape probability of the neutron-induced reaction products $\left({ }^{4} \mathrm{He}\right.$ and ${ }^{7} \mathrm{Li}$ ions $)$ into the drift gap. The total detection efficiency is given by the product of these two factors, assuming that all the ${ }^{4} \mathrm{He}$ and ${ }^{7} \mathrm{Li}$ ions crossing the drift gap will produce a detectable amount of ionization charges. The exact experimental conditions (neutron spectra, etc.) and the detector setup $\left({ }^{10} \mathrm{~B}\right.$, layer, drift gap, gas filling, etc.) were modeled by the MCNP simulations, resulting in an intrinsic neutron detection efficiency of around $4.9 \%$. This value is in agreement with the detection efficiency calculated from experiments $(\sim 4.7 \%)$ considering that the counting rate measured during the acquisition of a flat field image (irradiation of the full detector active area, see Sec. III B), was of the order of around 710 counts $/ \mathrm{cm}^{2} / \mathrm{s}$, for an impinging neutron flux of about $1.5 \times 10^{4}$ neutron $/ \mathrm{cm}^{2} / \mathrm{s}$.

\section{B. Spatial inhomogeneity and compensation algorithm}

The spatial response (pixel-to-pixel) of an imaging system may vary as a consequence of different reasons, including local variations of detector efficiency and the spatial variation 


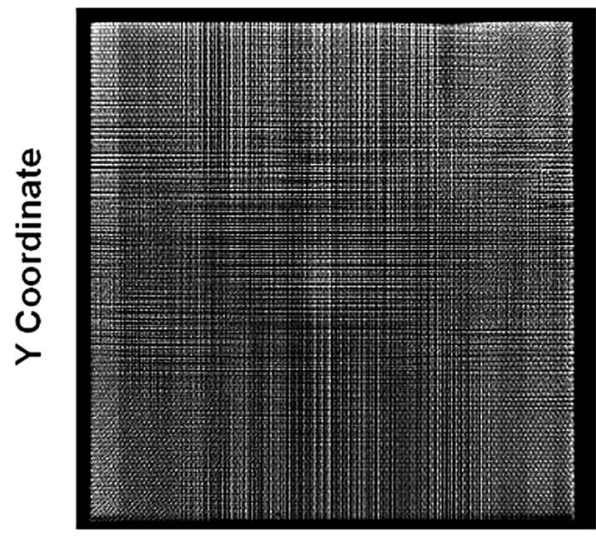

\section{$X$ Coordinate}

FIG. 2. Empty field image obtained by cold neutron irradiation (around 10 min of irradiation).

of the impinging neutron flux. The causes for local variations of sensitivity across the $10 \times 10 \mathrm{~cm}^{2}$ effective detector area may be due to the intrinsic efficiency variation of the different elements, including the neutron-to-charge particle conversion efficiency of the ${ }^{10} \mathrm{~B}$ layer (which mainly depends on the homogeneity as well as the thickness of the ${ }^{10} \mathrm{~B}$ layer); gain variation across the THGEM multipliers; and variation of the induced signal-transparency of the resistive layer before the X-Y pad electrodes (the resistive layer is made of graphite spread on a G10 substrate, with an average resistivity of $1 \mathrm{M} \Omega$ /square - variation of the graphite layer may result in variation of surface resistivity and thus signal-transparency). All these variations result in substantial high-frequency nonuniformities in the raw data image.

Figure 2 shows a radiographic image of the empty field obtained by cold neutron irradiation. The image was acquired for a total of $10 \mathrm{~min}$ of irradiation. The image is rather inhomogeneous and it is interesting to notice two peculiar patterns that emerge from it: on large scale, a continuous and regular series of stripes are clearly distinguishable in both the vertical and horizontal coordinates; on smaller scale, it is possible to distinguish small spots of high luminosity forming regular hexagonal patters. These latter structures perfectly matched the geometry and dimension of the THGEM holes pattern (Figure 3). This effect was also observed in a previous work ${ }^{26}$ and it proves that THGEM detector is capable of resolving the sub-millimeter granularity of the multiplier electrode. The intrinsic spatial resolution of the imaging system is then better than the $\sim 0.7 \mathrm{~mm}$ (FWHM) reported in a previous work ${ }^{23,26}$ under soft X-ray irradiation and the $1.3 \mathrm{~mm}$ FWHM calculated in this report with cold neutron (see Sec. III B).

Some interesting deeper evaluations on the imaging detector performance, in terms of homogeneity response and image noise, were obtained by the investigation of the empty field image of Figure 3 by means of Fourier analysis. We have calculated the projections of the image along the two coordinates (an example of $x$ coordinate projection is shown in Figure 4). The image projection in the $x$ coordinate (y coordinate) was obtained by integrating (summing) the luminosity (counts) of the pixels along the $y$ coordinate ( $\mathrm{x}$ coordinate).

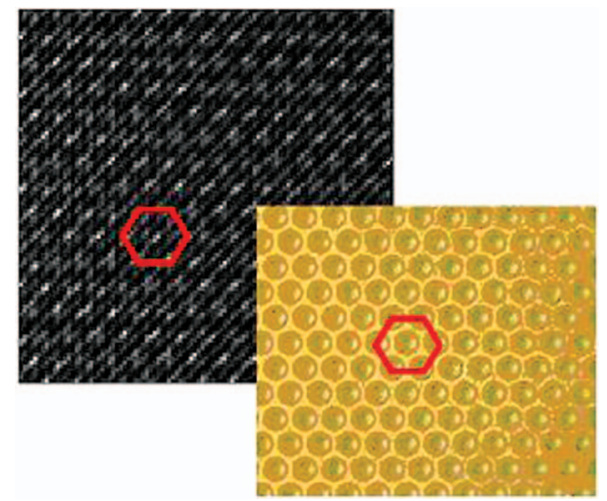

FIG. 3. From left to right: A zoom of a small area of the empty filed image (shown in Fig. 2), compared to a picture of the holes pattern on a THGEM surface. The structure of the hexagonal pattern of the THGEM hole is clearly visible also in the image.

The projection shows large variations of luminosity (noise) along the full detector length. The spectral analysis of these projections, by means of Fourier transform (FT), allows one to find those frequency components buried in the noisy image projection and reveals and characterizes possible regular structures in the spatial domain of the original image. FT of the two projection coordinates are shown in Figure 5. Two particular frequency components are clearly revealed, corresponding to $1 \mathrm{~mm}$ and $0.5 \mathrm{~mm}$ in the spatial domain. They correspond to the disposition of the THGEM holes: namely THGEM hole pitch $(1 \mathrm{~mm})$ and THGEM hole line alignment resulting from the hexagonal distribution of the holes $(0.5 \mathrm{~mm})$ (see Figure 6). Other frequencies in the two coordinates are due to the combination of the hexagonal hole patterns, resulting by the disposition of the two THGEM (one is rotated by $90^{\circ}$ with respect to the other).

It is shown that the main source of inhomogeneity affecting the image of the present THGEM detector system is due to the geometrical structure of the THGEM multiplier and the particular disposition of the THGEM holes, where the electron avalanche is taking place. Information of this pixel-topixel inhomogeneity response function can be used for the reconstruction of quantitative and artifact-free images. The

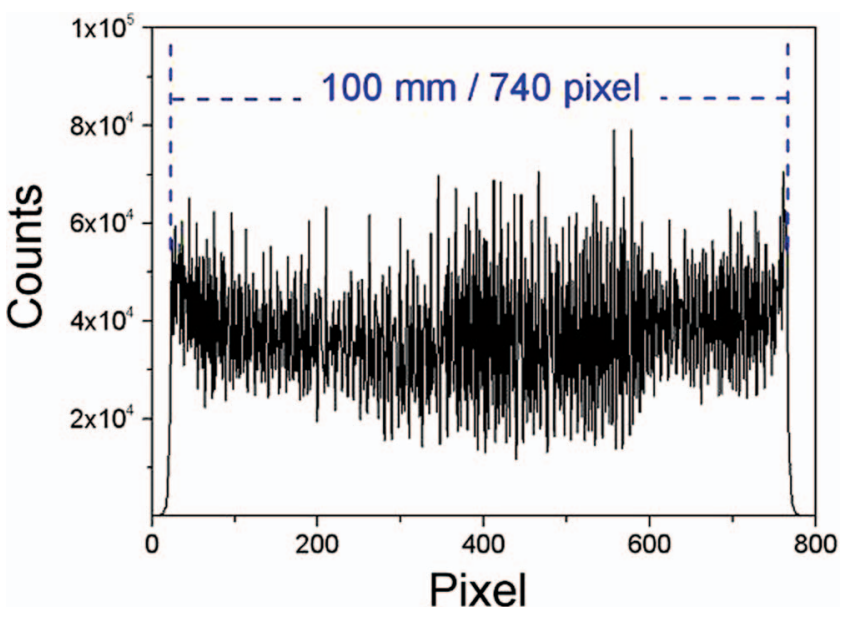

FIG. 4. Image projection along the x-coordinate. 

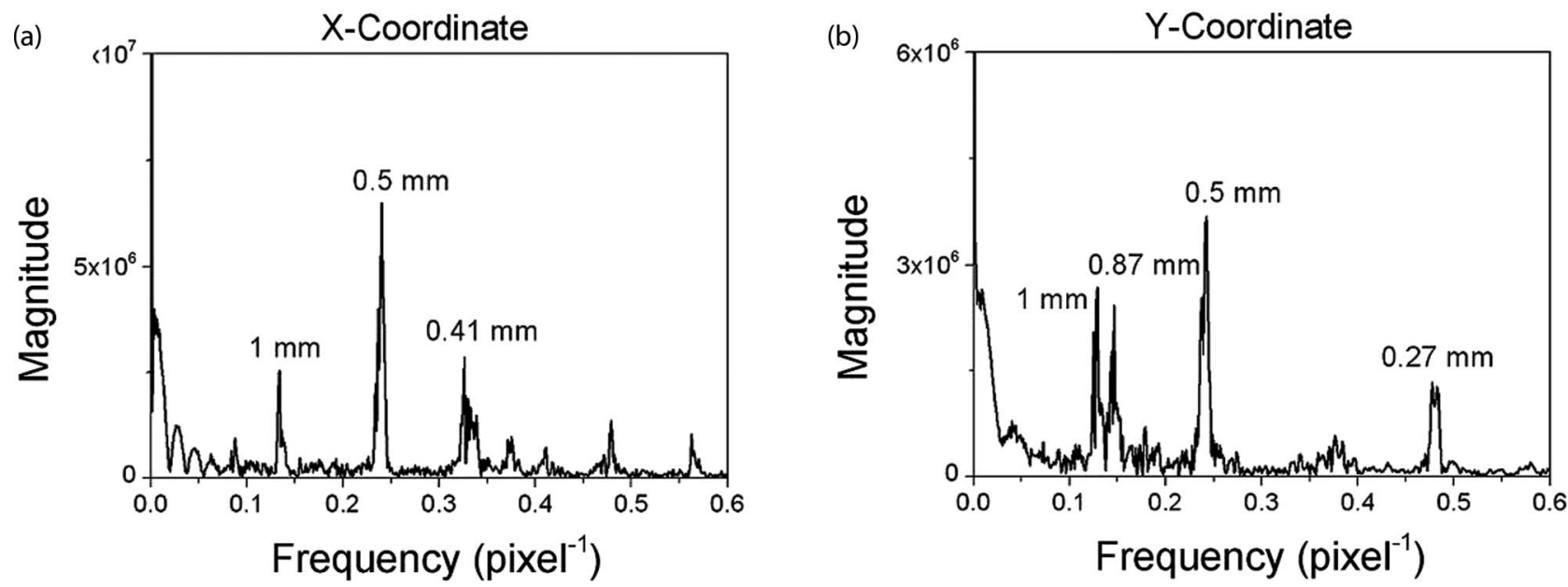

FIG. 5. Fourier transforms (FT) analysis of the projections (along the $\mathrm{x}$ and $\mathrm{y}$ coordinates, respectively, on the left and on the right graph) of the empty field image of Figure 2.

process of correcting for these effects is known as flat-field correction (FFC) and the individual correction factors for each pixel are referred to as flat-field correction coefficients. From a mathematical point of view, the FFC coefficient $C_{x, y}$ for the pixel in the position $(\mathrm{x}, \mathrm{y})$ are computed as

$$
\mathrm{C}_{\mathrm{x}, \mathrm{y}}=\frac{\mathrm{A}}{\mathrm{F}_{\mathrm{x}, \mathrm{y}}},
$$

where $F_{x, y}$ is the luminosity of the pixel $(x, y)$ in the flat-field image and $\mathrm{A}$ is the average value of the flat field luminosity calculated over the full active area.

Figure 7 shows examples of a cold-neutron radiographic images of two thin gadolinium (Gd) absorbers (a rectangular strip and circular patch), before (a) and after (b) FFC processing. Note that the FFC obviously also eliminates any nonuniformity originating from the beam intensity profile over the sensitive area of the detector. After the FFC the pixel-topixel variation in luminosity should be solely due to Poisson statistic. Figure 8 depicts the histogram of a small region of Figure 7(b), corresponding to an empty field (no object in the field of view). The spread of the histogram $(\sigma)$ is equal to the square root of the average luminosity, which is exactly the variance of a Poisson distribution.

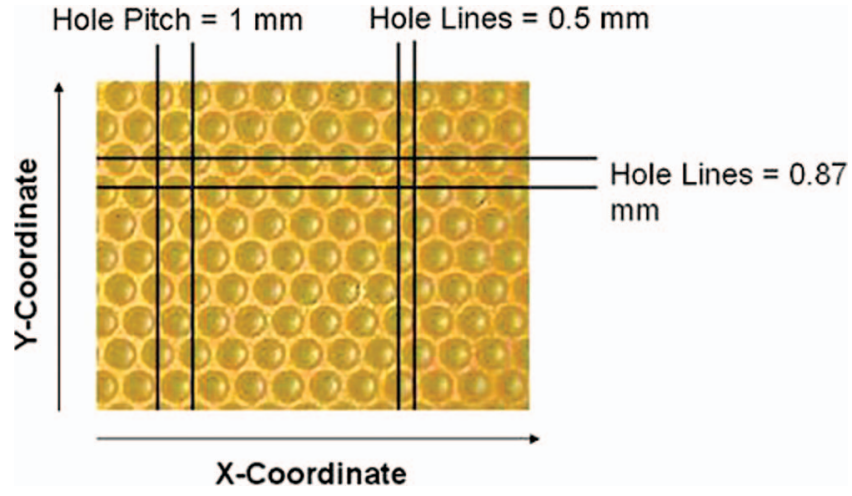

FIG. 6. Details of the hexagonal distribution of the THGEM holes. Hole pitch is $1 \mathrm{~mm}$ while the distance between the THGEM hole alignment resulting from the hexagonal pattern is $0.5 \mathrm{~mm}$. This geometrical pattern results in a specific noise distribution of the THGEM detector image.

\section{Spatial resolution}

The spatial resolution was derived using the edge spread function (ESF) technique, by the analysis of the FFCprocessed image of the gadolinium absorbers shown in Figure 9.

The ESF for both the $\mathrm{x}$ - and $\mathrm{y}$-coordinate was obtained by calculating the profile of two selected regions of the edge image of the rectangular-shaped Gd absorber (see Figure 9). The two profiles (black symbols in Figure 10) were then fitted with a suitable function assumed as the analytical ESF model; the curve fitting avoids amplifying the image noise during the derivative computation of the averaged over-sampled ESF. The calculation of the Line Spread Function (LSF) and the related spatial resolution (FWHM of the LSF) is then straightforward by analytically processing the model function. There are several different analytical function models for the ESF fitting, such as Cauchy function, Gumbel function, hyperbolic function, Laplace function, Gauss function, etc. In general the logistic (Fermi) function was found to the give the best result. The logistic (Fermi) function $\left(\mathrm{ESF}_{\mathrm{Fermi}}\right)$ can be expressed as

$$
\operatorname{ESF}_{\text {Fermi }}(x)=a+\frac{b}{1+\exp [-c(d-x)]},
$$

where $\mathrm{a}$ is the offset, $\mathrm{b}$ is the contrast, and $\mathrm{c}$ and $\mathrm{d}$ are the steepness and the centroid of the edge, respectively.

(a)
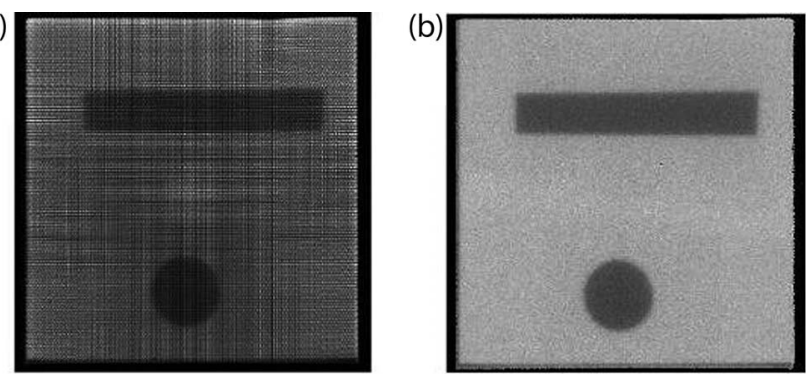

FIG. 7. Example of a cold-neutron radiographic image of two absorbers (made of gadolinium) before (a) and after (b) FFC processing. 


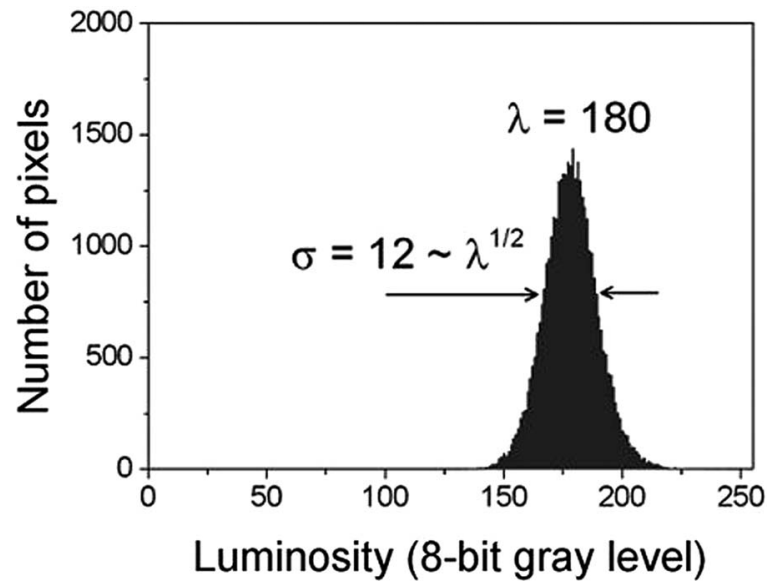

FIG. 8. Histogram of the central part (empty field) of the FFC-processed image in Figure 7(b). The spread of the distribution of pixel luminosity is due only to Poisson statistics.

As the two-dimensional derivative of an edge is a line, the LSF can be determined by taking the derivative of the ESF. Moreover, as the LSF may also be interpreted as the projection integral of the point spread function (PSF), the onedimensional derivative of the ESF corresponds to the PSF. By definition, the spatial resolution of the system can be expressed by the FWHM of the PSF, derived from the measured ESF of the system (blue graph in Figure 10). In particular, it is easy to show that for the logistic Fermi function model, the spatial resolution (FWHM) is calculated as

$$
\text { Spatial resolution }=\frac{3.53}{\mathrm{c}},
$$

where $\mathrm{c}$ is the steepness parameter of the logistic Fermi function (Eq. (3)). The spatial resolution of the THGEM-based imaging system results to be of $1.32 \mathrm{~mm}$ and $1.38 \mathrm{~mm}$, respectively, along the $\mathrm{x}$ - and $\mathrm{y}$ - coordinate. However, note that the analysis of the empty field image reveals details of the THGEM structure (see holes patter in shown in Figure 3) of sub-millimeter dimension, so that the intrinsic localization capability of the THGEM-imaging detector must be better then

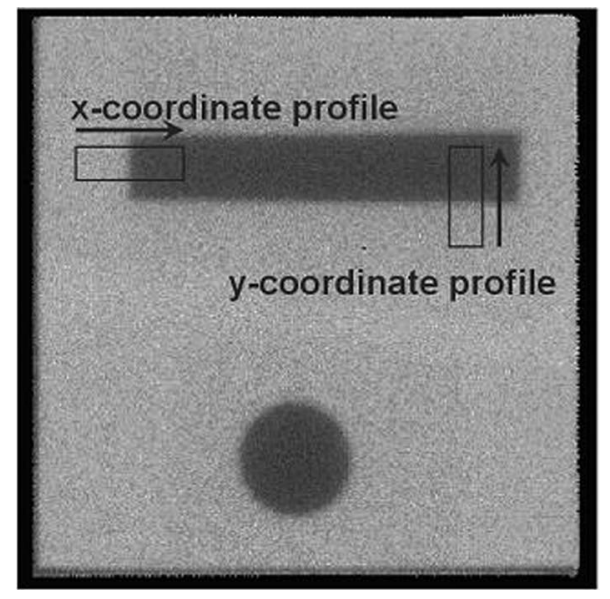

FIG. 9. Selected area of the Gd absorber image used to calculated the spatial resolution.

the measured 1.32-1.38 mm spatial resolution (FWHM). The further degradation of the localization properties of the full imaging system is then due to the spread of the ${ }^{10} \mathrm{~B}$ fission products (and resulting ionization) in the drift field.

Indeed, the spatial resolution of the entire cold-neutron imaging system $\left(\sigma_{\text {tot }}\right)$ may be written as the sum of two components

$$
\left(\sigma_{\text {tot }}\right)^{2}=\left(\sigma_{\text {converter }}\right)^{2}+\left(\sigma_{\text {THGEM }}\right)^{2},
$$

where $\sigma_{\text {converter }}$ is the spread of the ionization electron created by the ${ }^{10} \mathrm{~B}$ fission product $\left({ }^{4} \mathrm{He}\right.$ and $\left.{ }^{7} \mathrm{Li}\right)$ in the drift gap/field, while $\sigma_{\text {THGEM }}$ is the intrinsic localization capability of the THGEM imaging system.

Figure 11 shows the profile of the spatial distribution of the energy (and thus ionization electrons) deposited in the $1 \mathrm{~mm}$ gas gap $\left(\mathrm{Ne} / \mathrm{CF}_{4}\right)$, computed along the direction parallel to the converter foil. The calculation was performed using MCNPX, simulating the irradiation of the $1 \mu \mathrm{m} \mathrm{10B}$ converter layer with a infinitesimal small neutron beam. The energy deposited by the alpha particle (the other ${ }^{10} \mathrm{~B}$ fission product) shows a similar spatial distribution.
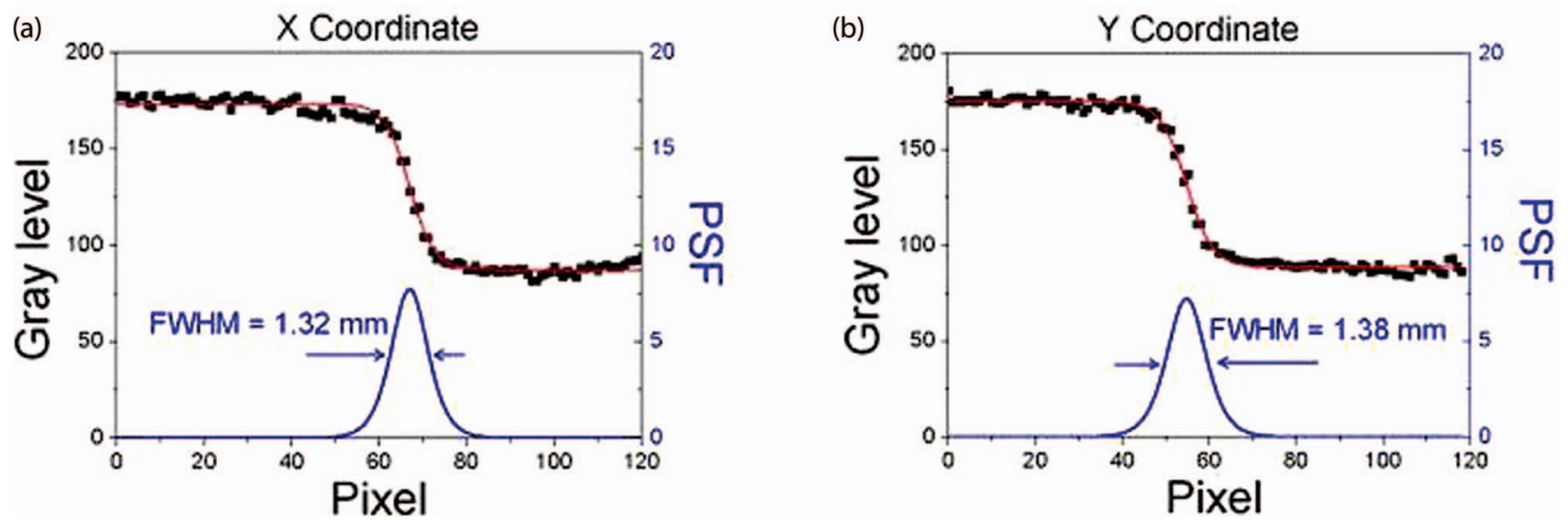

FIG. 10. ESF raw data (circle symbols) and the fitting (lines) using the mathematical model (logistic Fermi function), calculated from the analysis of two areas of Figure 9: (a) Depicted profile along the x coordinate while (b) shows the profiles along the y coordinate. The figures show also the PSF (lower graphs) calculated from the fitting model. For both coordinates, the spatial resolution (FWHM of the PSF) is approximately $1.3 \mathrm{~mm}$. 


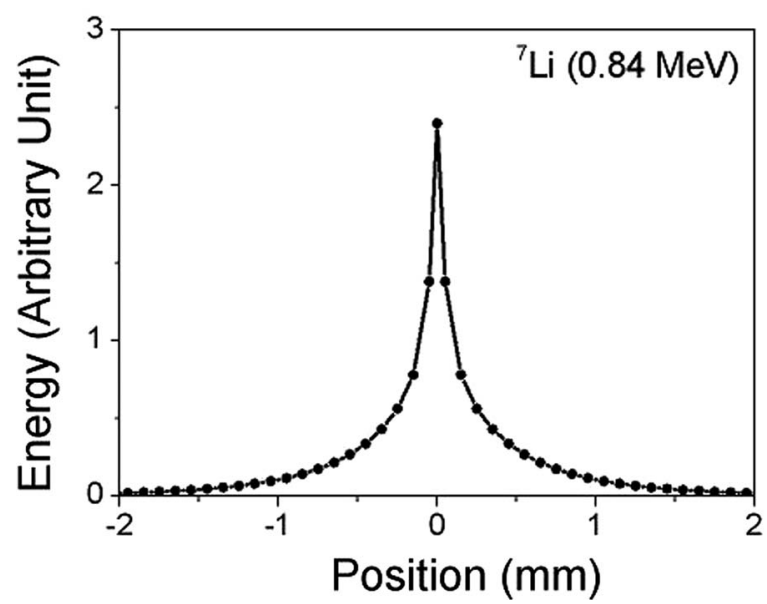

FIG. 11. Profile of the spatial distribution of the energy deposited by the $7 \mathrm{Li}$ $(0.84 \mathrm{MeV})$ in the $1 \mathrm{~mm}$ gas gap $\left(\mathrm{Ne} /(5 \%) \mathrm{CF}_{4}\right)$, computed along the direction parallel to the converter foil. The calculation was performed by MCNPX assuming an infinitesimal small neutron beam (pencil beam) impinging on the Boron-converter. Similar spatial distribution of deposited energy was found for the alpha particle.

It can easily be shown that the PSF $(\sim 1.35 \mathrm{~mm}$ FWHM $)$ obtained in Figure 10 can be obtained as the convolution of function describing the spatial distribution of deposited energy of the ${ }^{10} \mathrm{~B}$ fission product (depicted in Figure 11) and the intrinsic spatial resolution of the THGEM-imaging detector represented by a Gaussian function, with a FWHM of the order of $0.4 \mathrm{~mm}$. According to additional computer simulation (MCNP), better spatial resolution may be achieved by decreasing the size of the drift gap (with no substantial effects on THGEM detector performance, such as detection efficiency); for example, for a drift gap of $0.5 \mathrm{~mm}$ length, the

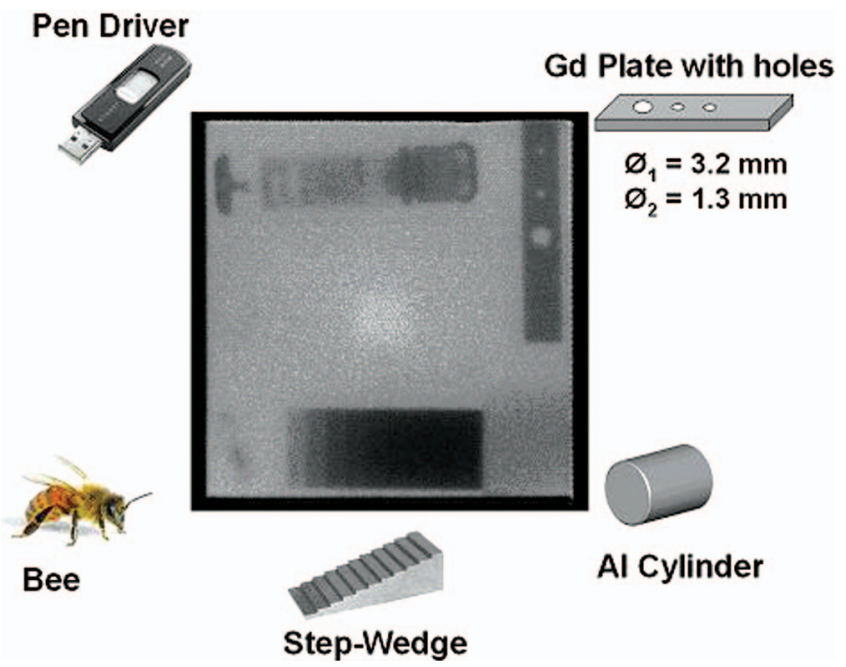

FIG. 12. Radiography image of various objects: the upper-left corner: pen driver, on the upper-right corner: a metal plate with holes of various diameters ( 3.2 and $1.3 \mathrm{~mm}$ ), lower-left corner: a bee, center: a brass step-wedge, and on the lower-right corner: an aluminum cylinder.

spread of the ionization distribution produced by the ${ }^{10} \mathrm{~B}$ fission products (Figure 11) would be reduced by around $30 \%$.

\section{Example of cold-neutron transmission image}

Figure 12 is an example of cold neutron transmission radiography image of various objects, including a pen driver, a Gd plate with holes (one hole of $3.2 \mathrm{~mm}$ diameter and two smaller holes of $1.3 \mathrm{~mm}$ diameter), a cylinder made of aluminum, a small insect (a bee), and a brass step-wedge (each step of $5 \mathrm{~mm}$ in surface and $5 \mathrm{~mm}$ rise).

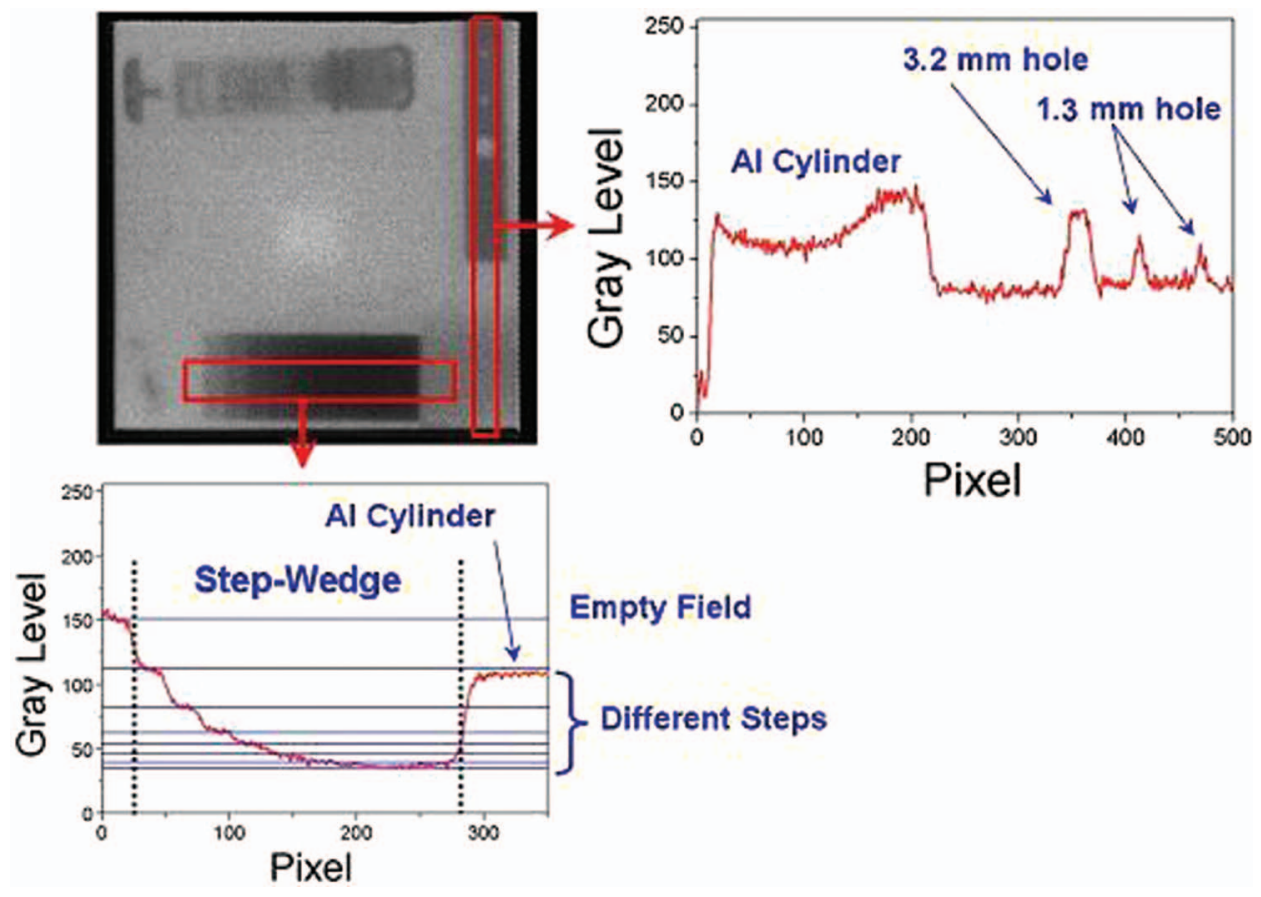

FIG. 13. Analysis (profiles calculation) of two areas of Figure 12 corresponding to the step-wedge image and image of the Al-cylinder plus Gd plate with holes of $3.2 \mathrm{~mm}$ and $1.3 \mathrm{~mm}$ diameters. In the two graphs, one pixel corresponds to $\sim 0.2 \mathrm{~mm}$. 
In order to illustrate the quality of the image, the profile of two interesting areas of Figure 12 were taken and analyzed. The graph in Figure 13(a) shows the profile of the step-wedge; the various steps with different thickness appear at different luminosity. From the analysis of the decreasing of the luminosity of the step as function of the thickness, we have calculated an effective attenuation coefficient for the cold spectrum at ICON of the order of $0.62 \mathrm{~cm}^{-1}$. Notice that the average wavelength of the ICON beam is around $34 \mathrm{~nm}$ (Kaestner et $a l .{ }^{20}$ ) which corresponds to a brass attenuation coefficient of about $0.65 \mathrm{~cm}^{-1}$, which is very close to the value obtained by the analysis of Figure 12. The graph in Figure 13(b) illustrates the profile of an $\mathrm{Al}$ cylinder $(4 \mathrm{~cm}$ diameter) and of a Gd bar with holes (one hole of $3.2 \mathrm{~mm}$ diameter and two holes of $1.3 \mathrm{~mm}$ diameter). Even for the small diameter hole, the contrast is still rather good and the two holes can be discerned.

\section{CONCLUSION AND OUTLOOK}

We have assembled and tested a cold-neutron imaging detection system using a thin ${ }^{10} \mathrm{~B}$ layer, for neutron-to-proton converter, coupled to a THGEM-based gaseous charge readout; the active area of the detector was of $10 \times 10 \mathrm{~cm}^{2}$. The detector operated in a fully stable operational condition (no sparks) for several hours, at a gain around $10^{4}$ in $\mathrm{Ne} /(5 \%) \mathrm{CF}_{4}$ at atmospheric pressure. The detection efficiency measured at the cold-neutron ICON beam was of around 5\%. Due to the geometrical structure of the THGEM multiplier (1 $\mathrm{mm}$ holes pitch) and of the charge read-out anode $(2 \mathrm{~mm}$ pitch between charge-pickup strips), the image of detector active area shows a large inhomogeneity that can be corrected using a suitable flat-filed correction algorithm. The spatial resolution of the imaging system is on the average around $1.35 \mathrm{~mm}$, but it could be improved by further reducing the drift length (distance between the neutron converter layer and the first THGEM electrode). The THGEMbased detectors are an attractive and low-cost solution for large-area imaging system; due to the ability of providing high counting rate capability $\left(\sim 10 \mathrm{MHz} / \mathrm{cm}^{2}\right),{ }^{27}$ they represent a realistic promising solution for achieving timing capabilities needed for pulsed neutron sources and time-resolved imaging.
${ }^{1}$ M. Strobl, I. Manke, N. Kardjilov, A. Hilger, M. Dawson, and J. Banhart, J. Phys. D: Appl. Phys. 42, 243001 (2009).

${ }^{2}$ N. Kardjilov, I. Manke, A. Hilger, M. Strobl, and J. Banhart, Mater. Today. 14, 248-256 (2011).

${ }^{3}$ Neutron Imaging and Applications, edited by I. S. Anderson, R. McGreevy, and H. Z. Bilheux, Neutron Application and Techniques (Springer Science + Business Media, LLC, 2009).

${ }^{4}$ W. J. Richards, J. R. Barrett, M. E. Springgate, and K. C. Shield, Appl. Radiat. Isot. 61, 675-682 (2004).

${ }^{5}$ P. Boillat, G. Frei, E. H. Lehmann, G. G. Scherer, and A. Wokaun, Electrochem. Solid-State Lett. 13, B25-B27 (2010).

${ }^{6}$ E. Deschler-Erb, E. H. Lehmann, L. Pernet, P. Vontobel, and S. Hartmann, Archaeometry 46, 647-661 (2004).

${ }^{7}$ M. Griesser, R. Traum, K. Vondrovec, P. Vontobel, and E. H. Lehmann, IOP Conf. Ser.: Mater. Sci. Eng. 37, 012011 (2012).

${ }^{8}$ T. T. Tominaga, F. A. M. Cássaro, K. Reichardt, O. O. S. Bacchi, J. C. M. Oliveira, and L. C. Timm, Appl. Radiat. Isot. 57, 369-374 (2002).

${ }^{9}$ M. Zarebanadkouki, Y. X. Kim, A. B. Moradi, H.-J. Vogel, A. Kaestner, and A. Carmina, Vadose Zone J. 11 (2012).

${ }^{10}$ J. L. Kickhofel, R. Zboray, M. Damsohn, A. Kaestner, E. H. Lehmann, and H.-M. Prasser, Nucl. Instrum. Methods Phys. Res. A 651, 297-304 (2011).

${ }^{11}$ R. Zboray, J. Kickhofel, M. Damsohn, and H. M. Prasser, Nucl. Eng. Des. 241, 3201-3215 (2011).

${ }^{12}$ E. H. Lehmann, A. Tremsin, C. Grunzweig, I. Johnson, P. Boillat, and L. Josic, JINST 6, C01050-C01050 (2011).

${ }^{13}$ A. S. Tremsin, J. B. McPhate, J. V. Vallerga, O. H. W. Siegmund, J. S. Hull, W. B. Feller, and E. H. Lehmann, Nucl. Instrum. Methods Phys. Res. A 604, 140-143 (2009).

${ }^{14}$ V. Radeka, N. A. Schaknowski, G. C. Smith, and B. Yu, Nucl. Instrum. Methods Phys. Res. A 419, 642-647 (1998).

${ }^{15}$ R. Alon, M. Cortesi, A. Breskin, and R. Chechik, JINST 3 P11001 (2008).

${ }^{16}$ S. Uno, M. Sekimoto, T. Murakami, M. Tanaka, S. Nakagawa, E. Nakano, F. Sugiyama, K. Nagaya, A. Sugiyama, and T. Uchida, IEEE Nucl. Sci. Symp. Conf. Rec. 6, 4623-4626 (2007).

${ }^{17}$ M. Klein and C. J. Schmidt, Nucl. Instrum. Methods Phys. Res. A 628, 9-18 (2011).

${ }^{18}$ M. Damsohn and H.-M. Prasser, Flow Meas. Instrum. 20, 1-14 (2009).

${ }^{19}$ A. Ylönen, W.-M. Bissels, H.-M. Prasser, Nucl. Eng. Des. 241, 2484-2493 (2011).

${ }^{20}$ A. P. Kaestner, S. Hartmann, G. Kühne, G. Frei, C. Grunzweig, L. Josic, F. Schmid, and E. H. Lehmann, Nucl. Instrum. Methods Phys. Res. A 659, 387-393 (2011).

${ }^{21}$ A. Breskin, R. Alon, M. Cortesi, R. Chechik, and J. Miyamoto, Nucl. Instrum. Methods Phys. Res. A 598, 107-111 (2009).

${ }^{22}$ M. Cortesi, V. Peskov, G. Bartesaghi, J. Miyamoto, S. Cohen, R. Chechik, J. M. Maia, J. M. F. dos Santos, G. Gambarini, V. Dangendorf, and A. Breskin, JINST 4, P08001-P08001 (2009).

${ }^{23}$ M. Cortesi, R. Alon, R. Chechik, A. Breskin, D. Vartsky, and V. Dangendorf, JINST 2, P09002 (2007).

${ }^{24}$ C. J. Borkowski and M. K. Kopp, Rev. Sci. Instrum. 46, 951-962 (1975).

${ }^{25}$ F. Brown, B. Kiedrowski, and J. Bull, MCNP5 Release Notes, LA-UR-1006235 (2010).

${ }^{26}$ G. Bartesaghi, Ph.D. dissertation, Università degli Studi di Milano and INFN, 2010.

${ }^{27}$ V. Peskov, M. Cortesi, R. Chechik, and A. Breskin, JINST 5, P11004 (2010). 